\title{
Changes in Bone Metabolism and Antioxidant Status with Combined Exercise and Honey Supplementation in Young Female Rats
}

\author{
Somayeh Sadat Tavafzadeh ${ }^{1}$, Foong Kiew 0oi ${ }^{1 *}$, Chee Keong Chen ${ }^{1}$ and Siti Amrah Sulaiman ${ }^{2}$ \\ ${ }^{1}$ Sports Science Unit, School of Medical Sciences, Universiti Sains Malaysia, Kelantan, Malaysia \\ ${ }^{2}$ Department of Pharmacology, School of Medical Sciences, Universiti Sains Malaysia, Kelantan, Malaysia
}

Received: April 01, 2015; Accepted: May 12, 2015; Published: June 01, 2015

*Corresponding author: Foong Kiew Ooi, Sports Science Unit, School of Medical Sciences, Universiti Sains Malaysia, 16150, Kubang Kerian, Kelantan, Malaysia, Tel: +609-767 6931; Fax: +609-764 1945; E-mail:fkooi@usm.my

\begin{abstract}
Purpose: To investigate the effects of combined jumping exercise and honey supplementation on bone metabolism and antioxidant status in female rats.

Methods: Forty eight 12-week old Sprague Dawley female rats were divided into four groups: control group (C), honey group $(\mathrm{H})$, jumping group (J), and combined jumping and honey group (JH). Jumping exercise consisted of 40 jumps/day for 5 days/week at a height of $40 \mathrm{~cm}$. Honey was given to the rats at a dosage of $1 \mathrm{~g} / \mathrm{kg}$ body weight/rat/day via force feeding for 7 days/week. At the end of the study, tibial proximal volumetric total Bone Mineral Density (BMD) and volumetric trabecular BMD, serum total calcium, serum Alkaline Phosphatase (ALP) (bone formation marker), serum C-terminal telopeptide of type 1 procollagen (1CTP) (bone resorption marker), serum $\mathrm{F}_{2}$-isoprostanes (oxidative stress marker) and serum Total Antioxidant Status (TAS) were measured.
\end{abstract}

Results: There were no significant differences in tibial proximal volumetric total BMD and volumetric trabecular BMD among all the groups. Eight weeks of jumping exercise and honey supplementation resulted in statistically significant higher serum total calcium concentration in $\mathrm{JH}$ group as compared to $\mathrm{C}, \mathrm{H}$ and $\mathrm{J}$ groups $(p<$ 0.05). Serum ALP was significantly higher in J group as compared to C group $(p<0.05)$. On the other hand, serum 1CTP was significantly lower in JH as compared to the other three experimental groups. The concentration of $\mathrm{F}_{2}$-isoprostanes was not statistically different among all the groups. Serum TAS was significantly higher in JH and $\mathrm{H}$ groups as compared to $\mathrm{C}$ group $(p<0.05)$ respectively.

Conclusion: The results of the present study showed that 8 weeks of jumping exercise and honey supplementation significantly reduced bone resorption and enhanced antioxidant status, but did not seem to elevate oxidative stress marker.

Keywords: Bone metabolism markers; Antioxidant status; Jumping exercise; Honey supplementation; Rats

\section{Introduction}

Physical activity is reported as one of the strategies for maximizing bone mass during early age and subsequently minimizing bone loss later in life $[1,2]$. The effect of physical activity is through modification in bone turnover which occurs through two fundamentally different processes, i.e. modeling and remodeling with the activities of osteoblast and osteoclast cells in the mechanisms of bone formation and bone resorption [3]. A number of biochemical assays have been described for measuring serum and urinary concentration of markers of bone metabolism [4] that allow a good estimation of bone metabolic processes and have been established as useful parameters in assessing changes in bone turnover. To date, the mechanisms by which combined exercise and dietary intake lead to changes in bone metabolism are not fully understood.

Nutritional supplementation is another main factor associated with bone health and development. Aoe et al. [5] reported that as compared to drugs that have a rapid effect, administration of nutrients related to bone metabolism is relatively safe and inexpensive. To date, the effects of different types of supplementation such as calcium, vitamin D, milk, soy and soy protein on bone mass, turnover and mineral density have been reported [6-9]. One of the nutrients that has long tradition of use within various medical systems and prescribed for variety of uses from ancient time is honey. Ariefdjohan et al. [10] reported that honey has the potential to increase calcium absorption after 2 days of acute feeding in rats. In addition, Hussain et al. [11] reported that honey has similar effects with hormone replacement therapy on bone densitometry in postmenopausal women. Therefore, it was hypothesized that honey may play a role in increasing bone health.

Tualang honey is a wild, multi floral honey produced by Apis dorsata bees that form hives on the branches of giant trees named Tualang in the Malaysian rainforest, and it has been reported recently that ovariectomized rats that received Tualang honey showed greater improvements in trabecular bone structure than rats receiving calcium [12], implying the beneficial effects of Tualang honey supplementation on bone. Nevertheless to date, administration of Tualang honey in combination with exercise on bone health and antioxidant status in rats has not been investigated, thus the present study was proposed. 
Physical activity and its relationship with elevated oxygen consumption and free radical production has been established previously [13]. Exhausting exercise in rats may increase Reactive Oxygen Species (ROS) production over the capacity of antioxidant defenses [14]. Alessio et al. [15] reported the evidence of oxidative stress after both aerobic and isometric exercise by increasing lipid hydroperoxides and protein carbonyls. Radak et al. [16] found that a single bout of physical exercise induced formation of ROS and nitrogen species and the related oxidative damage in rats. On the other hand, regular training is known to increase the resistance against ROS induced lipid peroxidation, and decrease accumulation of oxidative protein and DNA damage [17]. Furthermore, it is proposed that a bout of exercise may elevate the inherent capacity of the protective endogenous antioxidant system [18] and may lead to a protective adaptation in antioxidant system against the stress of physical activity.

Endogenous antioxidant system or enzymatic antioxidants such as superoxide dismutase, catalase and glutathione peroxidase, in conjunction with exogenous antioxidants consumed through dietary sources, such as vitamin A, vitamin C, vitamin $\mathrm{E}$, flavonoids and several micronutrients including iron, copper, zinc, selenium and manganese can play important roles in antioxidant system. The antioxidant property of the food can protect living organisms from oxidative damage, resulting in prevention of various diseases [19]. Food that are expected to have antioxidant properties are soybeans, oats, tomatoes and green tea. Honey also contains antioxidant properties and it has been studied recently due to its wide range of therapeutic functions.

Honey is considered as a part of traditional medicine. Long duration of storage without becoming spoiled and high osmotic pressure of honey which increase the resistance to spoilage by microorganism make honey a strong antibacterial nutrient. Besides that, honey contains a number of components known to act as preservatives. These include $\alpha$-tocopherol, ascorbic acid, flavonoids and other phenolics and enzymes such as glucose oxidase, catalase and peroxidase [19] which all are known to have antioxidant activities. A number of studies reported the antioxidant activity of different types of honey from various regions and floral sources [19,20]. The composition of a particular honey sample greatly depends on the composition of nectar, where it originates, the method used to collect the nectar, seasonal and environmental factors, and geographic origin, as well as storage conditions. Since the information on combined effects of a Malaysian honey and jumping exercise on one's bone turnover and oxidative stress marker are lacking, the present study was carried out to determine the changes in bone mineral density and metabolism, oxidative stress marker, and antioxidant status when jumping exercise is combined with Malaysian Tualang honey as a supplementation in the young female rats. In addition, the present study aimed to explore the antioxidant effect of honey to prevent the oxidative stress which may be caused by jumping exercise and at the same time to evaluate the additive effects of combined jumping exercise and honey on bone metabolism.

\section{Materials and Methods}

\section{Animals}

Forty eight eleven-week old Sprague-Dawley female rats were placed in the experimental room to acclimatize to the environment for a period of seven days. After one week of acclimatization, the rats were weighed for their initial body mass. Then the rats were block-randomized into four initial bodymass-matched groups with 12 rats per group (6 rats per cage). Throughout the study, the environment was maintained under constant temperature of $26-29{ }^{\circ} \mathrm{C}$ and relative humidity of 70 $75 \%$. The rats were exposed to a constant 12:12 light/dark cycle, with the light period starting from 7.00 p.m. to 7.00 a.m. for the entire experimental period. The reversed light/dark cycle, was implemented to allow jump training during the day. At the end of the experimental period, the rats were weighed once again to obtain the final body mass. Then they were anaesthetized, one at the time, by placing them for 2-3 minutes in a desiccated jar containing a chloroform-soaked gauzed pad before being decapitated using a small guillotine (Scientific Research Instrument, UK). Blood was collected from the decapitated site for further serum bone metabolism and oxidative stress markers, and serum total antioxidant status measurements. The research protocol was approved by the Animal Research Committee, USM, No.: USM/Animal Ethics Approval/2008/(39)(121).

\section{Animal's grouping}

Rats with initial body mass ranged 190-220 grams were block randomized into four groups, i.e., 8 weeks of sedentary without supplementation control (C), 8 weeks of honey supplementation without exercise $(\mathrm{H}), 8$ weeks of jumping exercise without honey supplementation (J), and 8 weeks of combined jumping exercise and honey supplementation $(\mathrm{JH})$. At the end of the study, the rats were weighed once again to obtain the final body weight. All the rats survived during the whole experimental period.

\section{Training programme}

Rats in the jumping group and combined jumping and honey supplementation group were trained to jump using a previously described protocol [21]. Jumping exercises were carried out from 8:30 a.m. to 11:30 a.m. Each rat was subjected to the exercise for 5 minutes duration per day for 5 days per week. Each rat was placed in a specially designed wooden box, measuring $30.5 \mathrm{x}$ $30.5 \times 40 \mathrm{~cm}$ in length, width and height respectively, and with a copper strip base that formed an electrical grid. The jumping exercise was initiated by applying electrical stimulation to the base of the box through a stimulator (Grass S48, U.S.A.). The stimulator was set to automatically deliver a stimulus of 50-80 $\mathrm{V}$ for 1 second and at 3 second intervals. To begin the exercise session, the rats were placed on the electrical grip with the stimulator turned off. When the stimulator was turned on, the rats jumped from the floor of the box to catch the top edge of the box with their forepaws. Upon reaching the top, they were then immediately repositioned by hand to the floor of the box to repeat the procedure. The requirement for electrical stimulus decreased over time when the rats became accustomed to the jumping 
exercise. The jump training began with an initial jumping height of $20 \mathrm{~cm}$, after which the height was increased gradually to 40 $\mathrm{cm}$ by the third day. After a few days of training, the rats began to jump without electrical stimulation. The rats that refused to jump were stimulated by the low voltage of electrical stimulation. The sedentary rats in the control group (free cage activity) were not given any electrical stimulus. In order to mimic the stress induced by handling before and after jumping exercise, the sedentary rats were handled 5 days per week for 8 weeks.

\section{Honey supplementation}

Malaysian Tualang honey was orally supplemented to the rats at the dosage of $1 \mathrm{~g} / \mathrm{kg}$ body mass/rat/day for 8 weeks via force feeding (gavages), $30 \mathrm{~min}$ prior to the jumping exercise. Body mass of the rats was measured biweekly, and the dosage of honey was calculated based on the most recent body mass measurement [22].

\section{Measurement of bone mineral density using peripheral Quantitative Computed Tomography (pQCT)}

Immediately after the sacrificing procedure, the left hind leg tibias of the rats were dissected. The effects of jumping on bone in these animals depend on forces generated by the hind limbs, thus the hind limb tibias were examined in this study [21]. After removal of the flesh from the tibia bones, they were wrapped in saline-soaked gauze pads to prevent dehydration and put into labeled plastic bags and stored at $-80{ }^{\circ} \mathrm{C}$ (Heto Ultra Freezer 3410 , Denmark) for subsequent measurements at the later date [21]. On the day of bone densitometry measurements, the tibias were fixed with a custom-made plastic holder for rats' tibias. The proximal and mid shaft regions of the tibias were then scanned at a resolution of $220 \mu \mathrm{m}$ (pixel size) using a multilayer pQCT (Densiscan 2000, Scanco Medical, Bassersdorf, Switzerland). The thickness of each layer was $1 \mathrm{~mm}$. The contours of the periosteum and endosteum were both identified using the built-in software programme for measuring densitometry parameters including proximal volumetric total BMD of the integral region inside periosteum $\left(\mathrm{g} / \mathrm{cm}^{3}\right)$, and volumetric BMD of trabecular region inside endosteum $\left(\mathrm{g} / \mathrm{cm}^{3}\right)$.

\section{Biochemical analysis}

Blood was collected from the decapitated site into a $10 \mathrm{ml}$ test-tube through a funnel and left aside for one hour to ensure complete clotting. Serum was then obtained by centrifugation (15 minutes, $4000 \mathrm{RPM}, 4{ }^{\circ} \mathrm{C}$; Health-Ratina 46RS, Germany), after which the serum was divided into five equal portions $(\sim 0.5$ ml per portion) and stored at $-80{ }^{\circ} \mathrm{C}$ until subsequent analysis. Serum total calcium was analyzed colorimetrically (Hitachi Automatic Analyzer 912, Bohringer Mannheim, Germany) using commercially available reagent kits (Randox, UK). Serum ALP was analyzed using an automatic analyzer (Hitachi Automatic Analyzer 912, Bohringer Mannheim, Germany) with commercially available reagent kits (Randox, UK). Serum C-terminal telopeptide of type 1 procollagen (1CTP) was analyzed by enzyme immunoassay kit (Creative Dignostics, ELISA, USA), and the concentration was determined by a photometric microplate reader (Molecular Devices; Versamax tunable microplate reader, USA).

Serum $\mathrm{F}_{2}$-isoprostanes was analyzed using an EnzymeLinked Immunosorbent Assay (ELISA) method and commercially available enzyme immunoassay kit (Cayman's 8-Isoprostanes EIA, USA), and the concentration was determined by a photometric microplate reader (Molecular Devices; Versamax tunable microplate reader, USA). Serum TAS was measured using a commercially available antioxidant assay kit (Randox, UK). Serum TAS of the serum was determined by the method based on the absorbance of ABTS $® \bullet+$ radical cation (2, 2'-Azino-di-[3ethylbenzthiazoline sulphonate]).

\section{Statistical analysis}

Statistical Package for Social Sciences (SPSS) version 20.0 was used for the statistical analysis. All the data are reported as mean \pm Standard Deviation (SD). After checking normality and homogeneity, the data with normal distribution and homogenous variances were analyzed using one-way Analysis Of Variance (ANOVA) to determine the significance of the difference between groups. When the One-way ANOVA revealed a significant difference, post hoc (least significant differences test) was used to determine the differences between specific means. The $P$ value of $<0.05$ was considered as statistically significant and used for all the comparisons.

\section{Results}

In the present study, there were no significant differences in initial body weight between all the experimental groups. The initial body weight of the rats in $\mathrm{C}, \mathrm{H}, \mathrm{J}$ and JH was $200.2 \pm 18.0$ g, $205.6 \pm 12.4 \mathrm{~g}, 200.4 \pm 15.2 \mathrm{~g}$ and $201.1 \pm 11.1 \mathrm{~g}$ respectively. Additionally, no significant differences were found in the final body weight of the rats among the groups, where the final body weight of the rats in C, H, J and JH was $227.6 \pm 14.6 \mathrm{~g}, 232.9 \pm 18.1$ g, $226.1 \pm 16.5 \mathrm{~g}$ and $224.7 \pm 13.8 \mathrm{~g}$ respectively.

The present study found that there were no significant differences in tibial proximal volumetric total bone mineral density and volumetric trabecular bone mineral density among all the groups. The value of tibial proximal volumetric total bone mineral density of $\mathrm{C}, \mathrm{H}, \mathrm{J}$ and $\mathrm{JH}$ was $0.90 \pm 0.15 \mathrm{~g} . \mathrm{cm}^{-3}, 0.92 \pm 0.17$ g.cm ${ }^{-3}, 0.93 \pm 0.06$ g.cm ${ }^{-3}$, and $0.98 \pm 0.12{\mathrm{~g} . \mathrm{cm}^{-3}}^{-3}$ respectively. The value of volumetric trabecular bone mineral density of $\mathrm{C}, \mathrm{H}$, J and $\mathrm{JH}$ was $0.90 \pm 0.19 \mathrm{~g} . \mathrm{cm}^{-3}, 0.91 \pm 0.20 \mathrm{~g} . \mathrm{cm}^{-3}, 0.91 \pm 0.09 \mathrm{~g} . \mathrm{cm}^{-3}$ and $0.96 \pm 0.13$ g.cm ${ }^{-3}$ respectively.

Eight weeks of jumping exercise combined with honey supplementation $(\mathrm{JH})$ elicited significantly higher $(p<0.05)$ serum total calcium concentration compared to three other groups as illustrated in Figure 1. Serum ALP was significantly higher $(p<$ 0.05 ) in J group as compared to the $\mathrm{C}$ and $\mathrm{H}$ groups respectively (Table 1). On the other hand, serum C-terminal telopeptide of type 1 procollagen (1CTP) which is a bone resorption marker was significantly lower in JH group as compared to $\mathrm{C}, \mathrm{H}$ and J groups respectively. There was also a lower level of 1CTP ( $p<$ 0.05 ) in the J group when compared with the $\mathrm{C}$ group (Figure 2). 


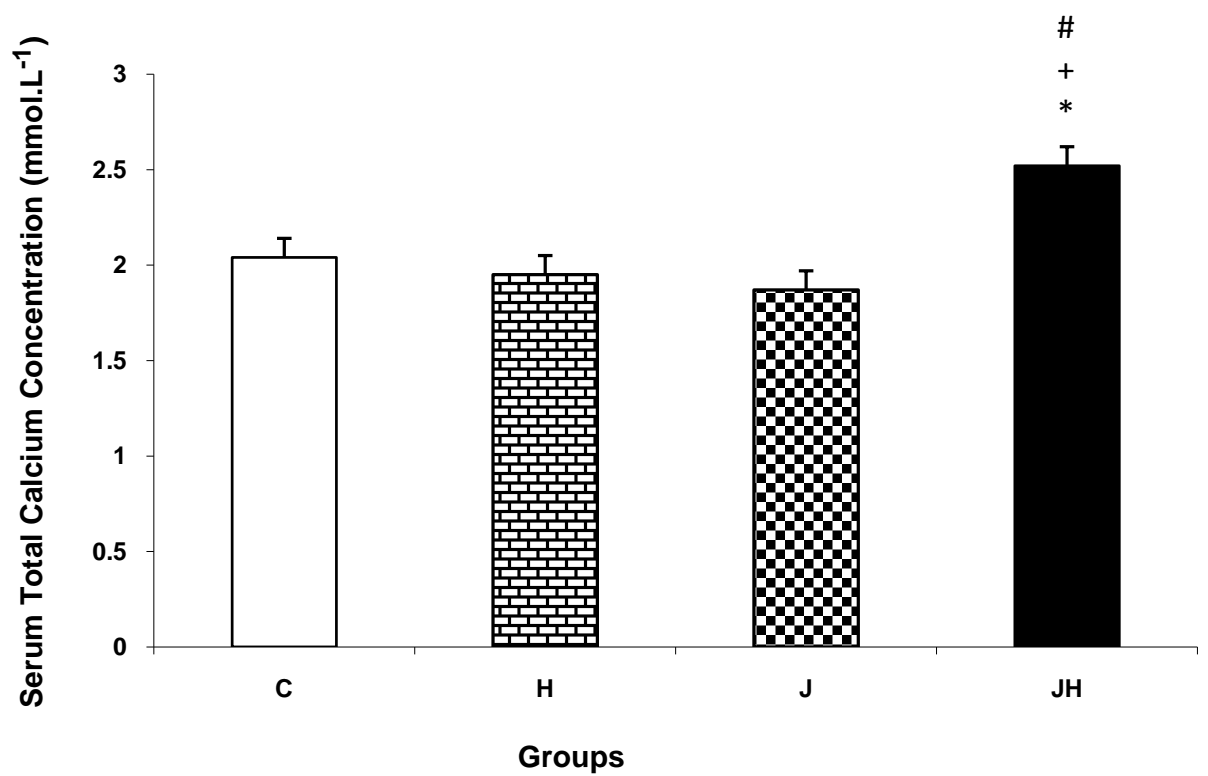

Figure 1: Mean serum total calcium concentration in the rats.

C: Sedentary without honey supplementation and jumping exercise control; H: Honey supplementation with $1 \mathrm{~g} / \mathrm{kg}$ body weight/day; J: Jumping exercise consisted of 40 jumps/day; JH: Honey supplementation combined with jumping exercise; *significantly different from control group (C) ( $p<$ $0.05)$; ${ }^{+}$significantly different from honey supplementation group $(\mathrm{H})(p<0.05)$; ${ }^{*}$ significantly different from jumping group (J) $(p<0.05)$.

Table 1: Mean serum alkaline phosphatase concentration in the rats.

\begin{tabular}{|c|c|}
\hline Groups & Serum alkaline phosphatase concentration (mmol. $\mathbf{L}^{-\mathbf{1}}$ ) \\
\hline $\mathrm{C}$ & $65.50 \pm 19.47$ \\
\hline $\mathrm{H}$ & $61.67 \pm 12.01$ \\
\hline $\mathrm{J}$ & $87.58 \pm 30.43^{*},+$ \\
\hline $\mathrm{JH}$ & $73.92 \pm 15.18$ \\
\hline
\end{tabular}

C: Sedentary without honey supplementation and jumping exercise control; H: Honey supplementation with $1 \mathrm{~g} / \mathrm{kg}$ body weight/day; J: Jumping exercise consisted of 40 jumps/day; JH: Honey supplementation combined with jumping exercise; *significantly different from control group (C) ( $p<$ $0.05)$; ${ }^{+}$significantly different from honey supplementation group (H) $(p<0.05)$.

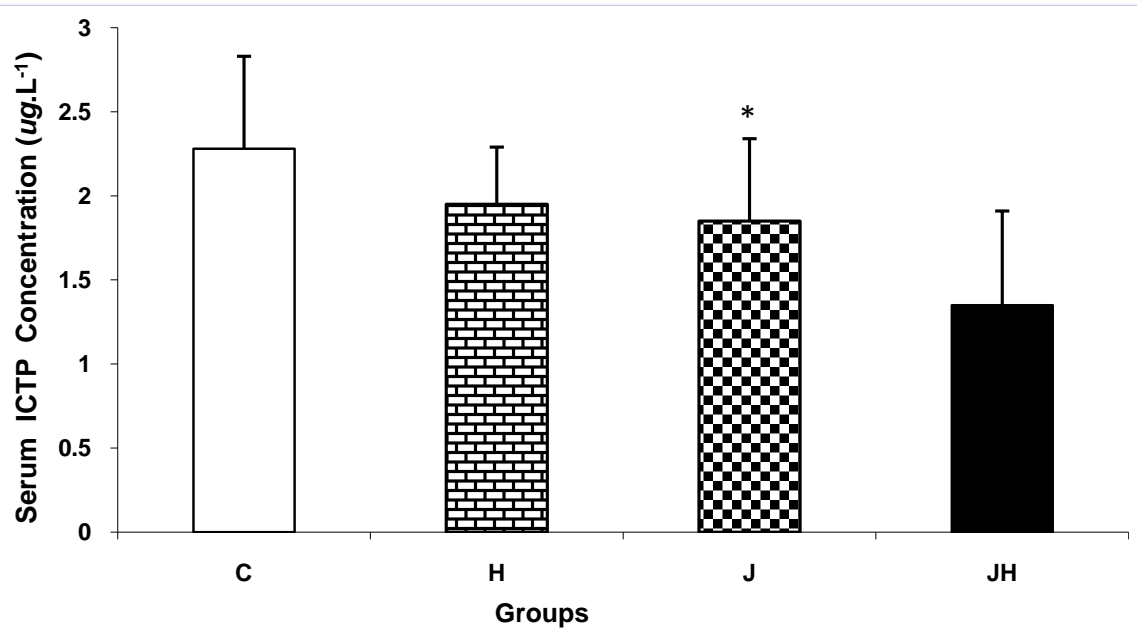

Figure 2: Mean serum C-terminal telopeptide of type 1 procollagen (1CTP) concentrations in the rats.

C: Sedentary without honey supplementation and jumping exercise control; H: Honey supplementation with $1 \mathrm{~g} / \mathrm{kg}$ body weight/day; J: Jumping exercise consisted of 40 jumps/day; JH: Honey supplementation combined with jumping exercise; *significantly different from control group (C) $(p<0.05) ;{ }^{* * *}$ significantly different from control group (C) $(p<0.001) ;{ }^{+}$significantly different from honey supplementation group (H) $(p<0.01)$; "significantly different from jumping group (J) $(p<0.05)$. 
In the $\mathrm{JH}$ group, the serum $\mathrm{F}_{2}$-isoprostanes, a marker of oxidative stress, was not significantly different from the J, $\mathrm{H}$ and C groups (Table 2). As shown in Figure 3, serum total antioxidant status of JH group was significantly higher as compared to the $\mathrm{C}$ group. The H group also demonstrated significantly higher serum total antioxidant status after 8 weeks of honey supplementation as compared to the $\mathrm{C}$ group $(p<0.05)$.

\section{Discussion}

In the present study, BMD, levels of serum bone metabolism and oxidative stress markers, and serum antioxidant status were evaluated in response to 8 weeks of jumping exercise (J), honey supplementation $(\mathrm{H})$ and combined jumping exercise with honey supplementation $(\mathrm{JH})$ as compared to sedentary non-supplemented control (C) in young female rats. The present study found that no significant differences were observed in tibial proximal volumetric total bone mineral density and volumetric trabecular bone mineral density among all the groups.

In serum total calcium, there was no significant difference in this measured parameter in $\mathrm{H}$ and J groups compared to $\mathrm{C}$ group. It was found that the level of serum total calcium was significantly higher in JH group than $\mathrm{C}, \mathrm{H}$ and J groups. Previous studies on the effects of exercise on serum calcium were inconsistent. Increase in serum calcium has been observed following physical activities
[23]. The absence of significant effects with jumping exercise alone on serum total calcium in the present study was consistent with Maimoun et al. [24] which reported that a single session of strenuous exercise did not result in any significant change in serum total calcium.

Honey has been reported to increase calcium absorption to the bone as a result of acute feeding in rats [10]. However, in the present study, increase in serum total calcium has been observed only when exercise was combined with honey supplementation. Similar observation was reported by Deyhim et al. [7] that there was no significant difference in serum total calcium of the rats that consumed isoflavones in comparison with those of sham and ovariectomized control groups. In an exercise combined with calcium supplementation study, Gala et al. [25] reported that serum calcium did not change in the ovariectomized rats performing running exercise with supplementation of calcium. However, it was also reported that swim training with and without soybean protein resulted in significant increases in plasma calcium concentrations compared to sedentary group in ovariectomized rats [26]. Similar finding of increased serum total calcium with combined honey supplementation and jumping exercised was reported in a recent study carried by the present research team [27]. The difference between the present study and Mosavat et al. [27] is that the prescribed jumping intensity in this

Table 2: Mean serum $\mathrm{F}_{2}$-isoprostanes concentration in the rats.

\begin{tabular}{|c|c|}
\hline Groups & Serum $\mathbf{F}_{2}$-isoprostanes concentration (pmol.L ${ }^{-1}$ ) \\
\hline C & $2047.70 \pm 696.89$ \\
\hline H & $1900.80 \pm 652.55$ \\
\hline$J$ & $1968.69 \pm 808.90$ \\
\hline$J H$ & $1419.60 \pm 539.52$ \\
\hline
\end{tabular}

C: Sedentary without honey supplementation and jumping exercise control; H: Honey supplementation with 1 g/kg body weight/day; J: Jumping exercise consisted of 40 jumps/day; JH: Honey supplementation combined with jumping exercise.

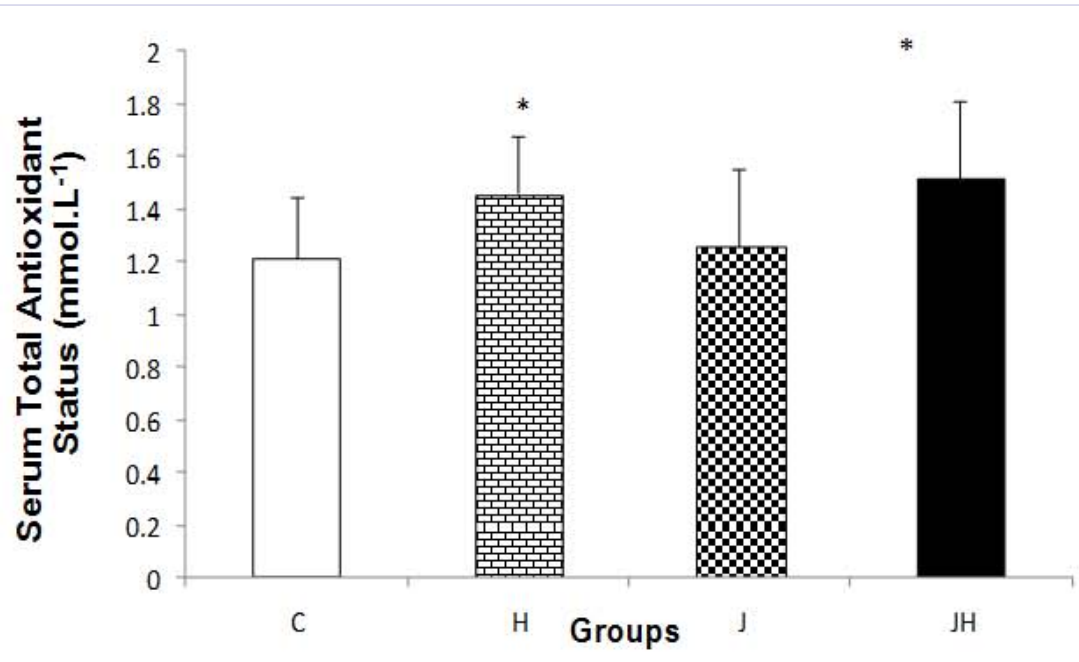

Figure 3: Mean serum total antioxidant status in the rats.

C: Sedentary without honey supplementation and jumping exercise control; H: Honey supplementation with 1 g/kg body weight/day; J: Jumping exercise consisted of 40 jumps/day; JH: Honey supplementation combined with jumping exercise; *significantly different from control group (C) ( $p$ $<0.05)$. 
previous study was 80 jumps per day, whereas the prescribed jumping intensity in the present study was 40 jumps per day. The observation of increase in serum total calcium concentration in the present study could be due to the combined effects of honey and jumping exercise which enhance the calcium absorption from intestines, and consequently might have augmented calcium retention in the bone.

Physical activities have been reported to increase bone formation markers and decrease bone resorption markers in animals as well as humans [28-31]. In the present study, there was higher level of serum ALP concentration, a bone formation marker, and lower level of serum C-terminal telopeptide of type 1 procollagen (1CTP), a bone resorption marker, in jumping exercise group as compared to control group. It seems that 8 weeks of jumping exercise (J) at the intensity of 40 times per day and height of $40 \mathrm{~cm}$, elicited beneficial effects on bone by increasing bone formation and reducing bone resorption. Honey supplementation alone $(\mathrm{H})$ did not affect serum ALP and 1CTP in this study. Interestingly, combination of jumping exercise and honey supplementation $(\mathrm{JH})$ did not show its effects on bone formation marker, however, significant lower level of 1CTP has been observed in $\mathrm{JH}$ as compared with $\mathrm{C}, \mathrm{H}$ and $\mathrm{J}$ groups. Maimoun et al. [32] also demonstrated that combined antioxidant supplementation and aerobic training did not alter urinary type 1CTP in elderly participants following 8 weeks of intervention. However, the reduction in bone resorption marker was similar to the study of Pasco et al. [33] who demonstrated that antioxidant supplements were associated with reduced serum 1CTP in nonsmoking post menopausal women.

As reported by Iwamoto et al. [29], bone gain through exercise in young rats was suggested to be attributed to increased bone formation reflected by ALP level and decreased bone resorption reflected by Tartrate-Resistant Acid Phosphatase (TRAP) in response to treadmill running exercise. Honda et al. [34] reported that eight weeks of jump training did not change serum 1CTP in ovariectomized rats. The inconsistent findings between Honda et al. [34] and the present study which showed that jumping alone (J) elicited lower value of 1CTP as compared to sedentary control may be due to the difference in exercise regimen, where the rats performed only 10 jumps per day in the previous study compared to 40 jumps per day in the present study. Ovariectomy may also contribute to the discrepancy between this previous study and the present study.

In studies which investigated the effects of supplementation on bone turnover, Elders et al. [35] reported that bone turnover reduced after 1 year of calcium supplementation, while no changes in markers of bone metabolism have been observed by Rosen et al. [36] after 1 year of calcium supplementation in early menopausal women. Supplementation of high soy protein is associated with lower level of bone resorption, but no changes in serum ALP in post-menopausal Japanese women as compared to calcium supplementation which had no influence on bone metabolism [37]. However, it was reported that consumption of isoflavones as a dietary supplementation did not influence serum ALP and TRAP activities in ovariectomized rats [7]. Similarly, in the present study, honey supplementation alone did not elicit any significant effect on bone turnover markers. However, one of the notable findings in the present study is that combination of jumping exercise and honey supplementation seem to reduce bone resorption significantly in the rats. Reduction in bone resorption marker of serum 1CTP was also observed in our recent study [27] with combined honey supplementation and jumping intensity of either 20 or 80 jumps per day. Due to its unique components, honey has been proven to have a variety of health benefits in humans. The main constituents of honey are carbohydrates such as monosaccharides, disaccharides, oligosaccharides, and polysaccharides as well as enzymes such as glucose oxidase, diastase, invertase, catalase, and peroxidase. Other chemical contents of honey are organic acid, ascorbic acid, vitamins, amino acids, flavonoids and phenolic acids [38]. Malaysian Tualang honey which has been used in our study is known for its antimicrobial, antiparasitic, antioxidant, and antiinflammatory effects. The high flavonoids and phenolic acid contents of Tualang honey has been proposed to have significant beneficial effects on bones [39]. Hardcastle etal. [40] reported that there is a significant association between total flavonoid intake and bone mineral density of hip and lumbar spine in a Scottish population. In the current study, the value of serum C-terminal telopeptide of type 1 procollagen (1CTP) was significantly lower in JH group as compared to all the other groups. The precise mechanism is unknown. However, it is speculated that the phenolic and flavonoid compounds in Tualang honey include kaempferol, quercetin, ellagic acid, gallic acid, hesperetin and catechin along with vital vitamins such as vitamin D, C, E and K and minerals when combined with exercise, could significantly inhibit the activity of osteoclast cells and consequently reduce bone resorption.

In terms of oxidative stress resulting from physical activity, studies have reported that physical exercise can induce a situation of oxidative stress [15]. In the present study, the level of $\mathrm{F}_{2}$-isoprostanes did not significantly differ among all the experimental groups. However, serum total antioxidant status was significantly higher in combined jumping exercise and honey supplementation group $(\mathrm{JH})$ as compared to the control group. These findings imply that this combination significantly enhanced antioxidant status, and therefore did not lead to any indication of oxidative stress. Similar finding of no change in $\mathrm{F}_{2}$-isoprostanes in response to exercise has been reported by Campbell et al. [41] that $\mathrm{F}_{2}$-isoprostanes did not significantly change in aerobic exercise intervention in a 12-month randomized, controlled trial study. In a study done by Yamamoto et al. [42], physically active rats which performed 6 weeks of voluntary running had shown an increase in antioxidant enzymes activities, such as Glutathione Peroxidase (GPx), Glutathione Reductase (GR) and Superoxide Dismutase (SOD).

Radak et al. [17] mentioned that the adaptation process in response to regular or habitual exercise and elevation in ROS formation can result in activation of the antioxidant system and affecting on oxidative damage repair systems, by increasing antioxidant enzymes activity, increasing resistance to oxidative stress, and subsequently lowering levels of oxidative damage. 
In the present study, the prescribed jumping exercise can be considered high-impact, short duration, consisting of 40 jumps per day for 8 weeks, did not exceed 5 minutes per day in comparison with long duration exhaustive exercises in the aforementioned studies. Probably this could be the reason for not observing any disturbance between antioxidant capacity and free radical production in the present study, while total antioxidant status significantly increased when exercise was combined with honey supplementation in JH group as compared to C group.

In the present study, $\mathrm{H}$ group demonstrated significantly higher level of serum total antioxidant status after 8 weeks of honey supplementation as compared to the control group. This observation could be due to Tualang honey which contains high antioxidant capacity. Antioxidant activity of different varieties of honey has been reported in a number of previous studies $[19,20,43,44]$. It has been shown that the antioxidant capacity of honey is strongly correlated with the total phenolic content relative to the amount of vitamins C and E [44]. Among several types of Malaysian honey, Tualang honey had the highest total phenolic and protein content compared to Gelam, Indian forest and Pineapple honey. Tualang honey has also been reported to have the highest ascorbic acid content which may be responsible for the elevated scavenging of ROS [45]. Furthermore, chronic exercise has been well documented to increase antioxidant defense mechanism via enhancement in antioxidant enzyme activity. Thus, honey intake which has been shown to have high antioxidant property combined with jumping exercise appears to have an additive effect on the total antioxidant status observed in this study.

7.The findings of the present study suggested that 8 weeks of jumping exercise in young female rats could elicit beneficial bone effect by increasing bone formation and reducing bone resorption. At the same time no sign of oxidative stress has been observed with this exercise protocol. When Malaysian Tualang honey was combined with jumping exercise, there were increment of serum total calcium, reduction of bone resorption and increased level of total antioxidant status. The increased total antioxidant status observed in this group could have ameliorated oxidative stress. However, further studies on the role of honey combined with exercise on the upregulation of both osteoclastic bone resorption and osteoblastic bone formation and antioxidant status in human subjects are warranted.

\section{Acknowledgments}

This work was supported by a 'Research University' grant from Universiti Sains Malaysia, Malaysia (Grant number: 1001/ PPSP/811079). We also would like to express our appreciation to Mdm. Jamaayah bt. Meor Osman, Mdm. Norlida bt Azalan, Mdm. Hafizah bt. Hamzah, Mdm Parimalah Velo and Mdm. Nor Aini bt. Sudin from Sports Science Unit, Universiti Sains Malaysia for their technical assistance.

\section{References}

1. Gómez-Cabello A, Ara I, González-Agüero A, Casajús JA, VicenteRodríguez G. Effects of training on bone mass in older adults: a systematic review. Sports Med. 2012; 42(4):301-25 doi:

\subsection{5/11597670-000000000-00000}

2. Mosti MP, Kaehler N, Stunes AK, Hoff J, Syversen U. Maximal strength training in postmenopausal women with osteoporosis or osteopenia. J Strength Cond Res. 2013; 27(10):2879-86. doi: 10.1519/ JSC.0b013e318280d4e2.

3. Allen MJ. Biochemical markers of bone metabolism in animals: uses and limitations. Vet Clin Pathol. 2003; 32(3):101-13.

4. Delmas PD, Eastell R, Garnero P, Seibel MJ, Stepan J. The use of biochemical markers of bone turnover in osteoporosis. Committee of Scientific Advisors of the International Osteoporosis Foundation. Osteoporos Int. 2000; 11 Suppl 6:S2-17.

5. Aoe S, Toba Y, Yamamura J, Kawakami H, Yahiro M, Kumegawa $\mathrm{M}$, et al. Controlled trial of the effects of milk basic protein (MBP) supplementation on bone metabolism in healthy adult women. Biosci Biotechnol Biochem. 2001; 65(4):913-8.

6. Prince R, Devine A, Dick I, Criddle A, Kerr D, Kent N, et al. The effects of calcium supplementation (milk powder or tablets) and exercise on bone density in postmenopausal women. J Bone Miner Res. 1995; 10(7):1068-1075.

7. Deyhim F, Stoecker BJ, Brusewitz GH, Arjmandi BH. The effects of estrogen depletion and isoflavones on bone metabolism in rats. Nutr Res. 2003; 23(1):123-30. doi:10.1016/S0271-5317(02)00477-3.

8. Ma DF, Qin LQ, Wang PY, Katoh R. Soy isoflavone intake inhibits bone resorption and stimulates bone formation in menopausal women: meta-analysis of randomized controlled trials. Eur J Clin Nutr. 2008; 62(2):155-61.

9. Tang BM, Eslick GD, Nowson C, Smith C, Bensoussan A. Use of calcium or calcium in combination with vitamin $\mathrm{D}$ supplementation to prevent fractures and bone loss in people aged 50 years and older: A metaanalysis. Lancet. 2007; 370(9588):657-66.

10. Ariefdjohan MW, Martin BR, Lachcik PJ, Weaver CM. Acute and chronic effects of honey and its carbohydrate constituents on calcium absorption in rats. J Agric Food Chem. 2008; 56(8):2649-54. doi: 10.1021/jf073357w.

11. Hussain NHN, Sulaiman SA, Hassan II, Kadir AA, Nor NM, Ismail SB, et al. Randomized controlled trial on the effects of Tualang honey and hormonal replacement therapy (HRT) on cardiovascular risk factors, hormonal profiles and bone density among postmenopausal women: A Pilot Study. J Food Res 2012; 1(2):171-88. DOI: 10.5539/ jfr.v1n2p171.

12.Zaid SSM, Sulaiman SA, Othman NH, Soelaiman IN, Shuid AN, Mohamad N, et al. Protective effects of Tualang honey on bone structure in experimental postmenopausal rats. Clinics(Sao Paulo). 2012; 67(7):779-84.

13. Ji LL. Antioxidants and oxidative stress in exercise. Proc Soc Exp Biol Med. 1999; 222(3):283-92.

14. Gul M, Atalay M, Hanninen O. Endurance training and glutathionedependent antioxidant defense mechanism in heart of the diabetic rats. J Sports Sci Med. 2003; 2(2):52-61

15. Alessio HM, Hagerman AE, Fulkerson BK, Ambrose J, Rice RE, Wiley RL. Generation of reactive oxygen species after exhaustive aerobic and isometric exercise. Med Sci Sports Exerc. 2000; 32(9):1576-81.

16. Radak Z, Sasvari M, Nyakas C, Kaneko T, Tahara S, Ohno H, et al. Single bout of exercise eliminates the immobilization-induced oxidative stress in rat brain. Neurochem Int. 2001; 39(1):33-8.

17. Radak Z, Chung HY, Goto S. Systemic adaptation to oxidative challenge 
induced by regular exercise. Free Radic Biol Med. 2008; 44(2):153-9. doi: 10.1016/j.freeradbiomed.2007.01.029.

18. Ohno H, Sato Y, Yamashita K, Doi R, Arai K, Kondo T, et al. The effect of brief physical exercise on free radical scavenging enzyme systems in human red blood cells. Can J Physiol Pharmacol. 1986; 64(9):1263-5.

19. Nagai T, Sakai M, Inoue R, Inoue H, Suzuki N. Antioxidative activities of some commercially honeys, royal jelly, and propolis. Food chem. 2001; 75(2): 237-40. doi:10.1016/S0308-8146(01)00193-5.

20. Schramm DD, Karim M, Schrader HR, Holt RR, Cardetti M, Keen CL. Honey with high levels of antioxidants can provide protection to healthy human subjects. J Agric Food Chem. 2003; 51(6):1732-5.

21. Ooi FK, Singh R, Singh HJ, Umemura Y. Minimum level of jumping exercise required to maintain exercise-induced bone gains in female rats. Osteoporos Int. 2009; 20(6):963-72. doi: 10.1007/s00198-0080760-6.

22. Tavafzadeh SS, Ooi FK, Krasilshchikov O, Siti AS. Effect of a combination of jumping exercise and honey supplementation on the mass, strength and physical dimensions of bones in young female rats. Journal of ApiProduct and ApiMedical Science. 2011; 3(1):26-32. Doi: 10.3896/ IBRA.4.03.1.05.

23. Brahm H, Piehl-Aulin K, Ljunghall S. Bone metabolism during exercise and recovery: the influence of plasma volume and physical fitness. Calcif Tissue Int. 1997; 61(3):192-8.

24. Maimoun L, Simar D, Malatesta D, Caillaud C, Peruchon E, Couret I, et al. Response of bone metabolism related hormones to a single session of strenuous exercise in active elderly subjects. Br J Sports Med. 2005; 39(8):497-502.

25. Gala J, Piedra CDL, Calero J. Short-and long-term effects of calcium and exercise on bone mineral density in ovariectomized rats. Br J Nutr. 2001; 86(4):521-7.

26. Figard H, Mougin F, Gaume V, Berthelot A. Combined intervention of dietary soybean proteins and swim training: effects on bone metabolism in ovariectomized rats. J Bone Miner Metab. 2006; 24(3):206-12.

27. Mosavat M, Ooi FK, Mohamed M. Effects of honey supplementation combined with different jumping exercise intensities on bone mass, serum bone metabolism markers and gonadotropins in female rats. BMC Complement Altern Med. 2014; 14(126):1-8. doi: 10.1186/14726882-14-126.

28. Fujimura R, Ashizawa N, Watanabe M, Mukai N, Amagai H, Fukubayashi $\mathrm{T}$, et al. Effect of resistance exercise training on bone formation and resorption in young male subjects assessed by biomarkers of bone metabolism. J Bone Miner Res. 1997; 12(4):656-62.

29. Iwamoto J, Shimamura C, Takeda T, Abe H, Ichimura S, Sato Y, et al Effects of treadmill exercise on bone mass, bone metabolism, and calciotropic hormones in young growing rats. J Bone Miner Metab. 2004; 22(1): 26-31.

30. Ooi FK, Ismail N, Abdullah MY. Effects of combined aerobic dance exercise and honey supplementation on bone turnover markers in young females. Asian Journal of Exercise and Sport Science. 2011; 8:111.

31. Kishimoto K, Lynch RP, Reiger J, Yingling VR. Short-term jump activity on bone metabolism in female college-aged-nonathletes. J Sports Sci Med. 2012; 11(1):31-8.

32. Maimoun L, Simar D, Caillaud C, Peruchon E, Sultan C, Rossi M, et al. Effect of antioxidants and exercise on bone metabolism. J Sports Sci. 2008; 26(3):251-8.

33. Pasco JA, Henry MJ, Wilkinson LK, Nicholson GC, Schneider HG, Kotowicz MA. Antioxidant vitamin supplements and markers of bone turnover in a community sample of nonsmoking women. J Womens Health (Larchmt). 2006; 15(3):295-300.

34. Honda A, Umemura Y, Nagasawa S. Effect of high-impact and lowrepetition training on bones in ovariectomized rats. J Bone Miner Res. 2001; 16(9):1688-93.

35. Elders PJ, Netelenbos JC, Lips P, Van Ginkel FC, Khoe E, Leeuwenkamp $\mathrm{OR}$, et al. Calcium supplementation reduces vertebral bone loss in perimenopausal women: a controlled trial in 248 women between 46 and 55 years of age. J Clin Endocrinol Metab. 1991; 73(3):533-40.

36. Rosen CJ, Chesnut CH 3rd, Mallinak NJ. The predictive value of biochemical markers of bone turnover for bone mineral density in early postmenopausal women treated with hormone replacement or calcium supplementation. J Clin Endocrinol Metab. 1997; 82(6):190410.

37. Horiuchi T, Onouchi T, Takahashi M, Ito H, Orimo H. Effect of soy protein on bone metabolism in postmenopausal Japanese women. Osteoporos Int. 2000; 11(8):721-4.

38. Khalil MI, Alam N, Moniruzzaman M, Sulaiman SA, Gan SH. Phenolic acid composition and antioxidant properties of Malaysian honeys. J Food Sci. 2011; 76(6):C921-8. doi: 10.1111/j.1750-3841.2011.02282.x.

39. Mohd Effendy N, Mohamed N, Muhammad N, Mohamad IN, Shuid AN. The effects of tualang honey on bone metabolism of postmenopausal women. Evid Based Complement Alternat Med. 2012;2012(938574):17. doi: $10.1155 / 2012 / 938574$.

40. Hardcastle AC, Aucott L, Reid DM, Macdonald HM. Associations between dietary flavonoid intakes and bone health in a Scottish population. J Bone Miner Res. 2011; 26(5):941-7. doi: 10.1002/ jbmr.285.

41. Campbell PT, Gross MD, Potter JD, Schmitz KH, Duggan C, McTiernan A, et al. Effect of exercise on oxidative stress: a 12-month randomized, controlled trial. Med Sci Sports Exerc. 2010; 42(8):1448-53. doi: 10.1249/MSS.0b013e3181cfc908.

42. Yamamoto T, Ohkuwa T, Itoh $\mathrm{H}$, Sato $\mathrm{Y}$, Naoi M. Effect of gender differences and voluntary exercise on antioxidant capacity in rats. Comp Biochem Physiol C Toxicol Pharmacol. 2002; 132(4):437-44.

43. Gheldof N, Wang XH, Engeseth NJ. Identification and quantification of antioxidant components of honeys from various floral sources. J Agric Food Chem. 2002; 50(21):5870-7.

44. Aljadi AM, Kamaruddin MY. Evaluation of the phenolic contents and antioxidant capacities of two Malaysian floral honeys. Food Chem. 2004; 85(4):513-8. doi:10.1016/S0308-8146(02)00596-4

45. Kishore RK, Halim AS, Syazana MS, Sirajudeen K. Tualang honey has higher phenolic content and greater radical scavenging activity compared with other honey sources. Nutr Res. 2011; 31(4):322-5. doi: 10.1016/j.nutres.2011.03.001. 